\title{
Distributed Privacy-Preserving Active Power Sharing and Frequency Regulation in Microgrids
}

\author{
Bo Fan, Member, IEEE, and Xiongfei Wang, Senior Member, IEEE
}

\begin{abstract}
To avoid potential privacy threats and associated cyber-security issues in microgrids, this letter presents a distributed active power sharing and frequency regulation method with preserved privacy of local information. In the proposed approach, the transmitted data including the active power outputs and capacities are protected by adding noises to the original ones. Theoretical analysis and verification studies are performed to illustrate the advantages of the proposed method.
\end{abstract}

Index Terms-Frequency regulation, power sharing, privacypreserving algorithm, distributed control.

\section{INTRODUCTION}

A MICROGRID generally comprises loads, distributed generators (DGs), and energy storage systems [1]. Usually, the DGs are connected to the microgrid through power electronic devices and regulated by hierarchical controllers to achieve various objectives including active power sharing and frequency regulation [2].

Traditionally, active power sharing is achieved by droop control. Centralized or distributed controllers are then utilized to compensate for the frequency deviations caused by the droop control [3]-[5]. However, the DGs' sensitive local data, such as power outputs, power capacities, utilization levels, etc., are directly transmitted through communication networks without privacy protection. In [6], a distributed optimal control is developed for microgrids to protect the DGs' privacy. Although the proposed method can preserve privacy to some extent, the accurate local frequency data is transmitted among the DGs during both the transient- and steady-state, which can be further used to infer the other local information, such as the real power generation of DGs.

To preserve the privacy of DGs, all information transmitted through the communication network should be protected. For this purpose, distributed average consensus algorithms are developed in [7], [8]. However, these algorithms are originally designed for ad-hoc networks where the data transmitted have no physical meanings. In microgrids, the transmitted data are essentially physical ones and have their physical impact. Hence, instability issues such as supply-demand imbalance may occur if these methods are directly applied. Besides, when using these methods, only average power sharing can be achieved. Since the DGs' capacities in microgrids are

Manuscript received September 18, 2020; revised January 5, 2021; accepted February 21, 2021. This work was supported in part by the Aalborg University Talent Management Program, and in part by the Marie Skłodowska-Curie Actions Individual Fellowships (H2020-MSCA-IF-2020) under Grant 101031512 (FRESLING). Paper no. PESL-00272-2020. (Corresponding author: Xiongfei Wang.)

B. Fan and X. Wang are with the Department of Energy Technology, Aalborg University, Aalborg 9220, Denmark (e-mail: bof@et.aau.dk; xwa@et.aau.dk).

(C) 2021 IEEE.

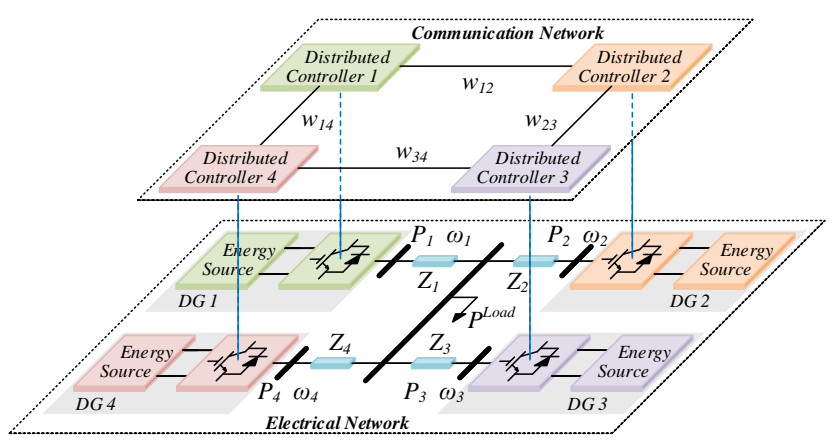

Fig. 1. Topology of a typical microgrid.

generally different from each other, the DGs with smaller capacities may be overloaded and the whole microgrid may suffer from the instability problem under the average power sharing scheme. To the best of the authors' knowledge, how to achieve the proportional active power sharing and frequency regulation in microgrids with preserved privacy of local information is still an open question.

To this end, this letter presents a distributed privacypreserving consensus (PPC)-based control method to achieve these control objectives. First, the original control problem is transformed into an equivalent active power reference generation problem which can be solved by obtaining the global active power utilization level. Further, a distributed PPC algorithm is proposed to acquire this global variable. With the noise added to the transmitted data, the privacy of DGs' local information is protected. Theoretical analyses illustrate that accurate proportional active power sharing and frequency regulation can be achieved. Finally, verification studies are conducted to demonstrate the merits of the proposed PPCbased method. Additionally, the presented methodology can be extended to other control problems in microgrids with preserved privacy.

The main contributions of this study are summarized as:

1) A distributed controller is firstly proposed to achieve the proportional active power sharing and frequency regulation in the microgrids with preserved privacy;

2) The transmitted data including the power outputs and capacities is protected by adding noises to the original ones. The instability impact that may be caused by using the traditional PPC-based methods is alleviated through the designed power reference generation technique and control structure;

3) Theoretical analyses and verification studies are performed to showcase the advantages of the proposed method. Both control objectives are proved to be accurately achieved in the steady-state. 


\section{Problem Formulation}

A typical cyber-physical microgrid composed of a communication network and an electrical one is illustrated in Fig. 1 [9]. The former is used for information sharing among the distributed controllers. The latter is a physical grid for delivering electrical energy from DGs to loads. Each DG is equipped with a distributed controller to generate the active power reference for its local droop controller.

For DG $i, i \in\{1,2, \ldots, N\} \triangleq \mathcal{V}_{E}$ with $N$ being the number of DGs, its local P-f droop control is expressed as

$$
\omega_{i}=\omega_{0}-m_{i}\left(P_{i}-P_{i}^{r}\right)
$$

where $\omega_{0}$ is the nominal frequency. $\omega_{i}$ is the DG's frequency. $P_{i}$ is the active power output, with $P_{i}^{r}$ being its reference. $m_{i}$ is the droop coefficient, which is selected properly to ensure the system stability when there exist equilibria [4], [5].

Two control objectives are considered in this study, i.e., the proportional active power sharing and frequency regulation [4], defined in the sequel.

Proportional Active Power Sharing is achieved if the total active power demand is shared proportionally according to DGs' capacities $P_{i}^{M}$ in the steady-state, i.e.,

$$
P_{i} / P_{i}^{M}=P_{j} / P_{j}^{M}, \quad \forall i, j \in \mathcal{V}_{E} .
$$

Usually, the proportional active power sharing is considered in microgrids to avoid the possible overload of the DG(s) when the total active power demand is no larger than the total capacity of the microgrid. Otherwise, load shedding must be taken to avoid the supply-demand imbalance.

Frequency Regulation is achieved if all the DGs' frequencies are equal to the nominal one in the steady-state, i.e.,

$$
\omega_{i}=\omega_{0}, \quad \forall i \in \mathcal{V}_{E}
$$

Additionally, the privacy of the local information, i.e., the active power outputs and capacities of DGs, is required to be protected during the realization of these objectives.

\section{Active Power RefErence Generation}

In this section, the control problem defined in Section II is transformed into an equivalent active power reference generation problem based on the global active power utilization level [1]. For a microgrid, the active power supply-demand balance can be expressed as $\sum_{i \in \mathcal{V}_{E}} P_{i}=P^{\text {Load }}+P^{\text {Loss }}$ where $P^{\text {Load }}$ and $P^{\text {Loss }}$ are the load active power requirement and the total active power loss of the transmission lines. Thereafter, the global active power utilization level $K^{U}$ can be defined as

$$
K^{U}=\frac{P^{\text {Load }}+P^{\text {Loss }}}{\sum_{i \in \mathcal{V}_{E}} P_{i}^{M}}=\frac{\sum_{i \in \mathcal{V}_{E}} P_{i}}{\sum_{i \in \mathcal{V}_{E}} P_{i}^{M}} .
$$

Moreover, select the active power references of the DGs' droop controllers as

$$
P_{i}^{r}=K^{U} P_{i}^{M}
$$

Next, the active power outputs and frequencies of DGs in the steady-state are analyzed based on the droop control (1) with the active power references given by (5).
Taking the summations of both sides of (5) and substituting (4) into the resulting equation yield

$$
\sum_{i \in \mathcal{V}_{E}} P_{i}^{r}=\frac{\sum_{i \in \mathcal{V}_{E}} P_{i}}{\sum_{i \in \mathcal{V}_{E}} P_{i}^{M}} \sum_{i \in \mathcal{V}_{E}} P_{i}^{M}=\sum_{i \in \mathcal{V}_{E}} P_{i}
$$

Since all the DGs' frequencies are identical in the steady-state, i.e., $\omega_{i}=\omega_{j}, \forall i, j \in \mathcal{V}_{E}$, combining (1) and (6) yields

$$
\begin{aligned}
& 0=\sum_{i \in \mathcal{V}_{E}} P_{i}^{r}-\sum_{i \in \mathcal{V}_{E}} P_{i}=\sum_{i \in \mathcal{V}_{E}}\left(P_{i}^{r}-P_{i}\right) \\
& =\sum_{i \in \mathcal{V}_{E}} m_{i}^{-1}\left(\omega_{i}-\omega_{0}\right)=\left(\omega_{i}-\omega_{0}\right) \sum_{i \in \mathcal{V}_{E}} m_{i}^{-1} .
\end{aligned}
$$

Considering the fact that the droop coefficients $m_{i}, \forall i \in \mathcal{V}_{E}$ are positive, one has $\sum_{i \in \mathcal{V}_{E}} m_{i}^{-1}>0$. Then (7) gives $\omega_{i}=$ $\omega_{0}, \quad \forall i \in \mathcal{V}_{E}$. Hence, all DGs' frequencies are equal to the nominal value $\omega_{0}$ in the steady-state. Furthermore, combining (1) and (5) gives $P_{i} / P_{i}^{M}=P_{i}^{r} / P_{i}^{M}=K^{U}, \quad \forall i \in \mathcal{V}_{E}$. Therefore, the ratios of all DGs' active outputs to their capacities are identical to the global utilization level.

From the above steady-state analysis, it can be noticed that if the global utilization level $K^{U}$ is known to all DGs, then the control objectives can be achieved simply by selecting the active power references of the droop control by (5). Besides, the active power supply-demand balance can be maintained. However, $K^{U}$ is a global variable whose calculation requires all the active power outputs and capacities of DGs. The privacy of these sensitive data is jeopardized.

\section{PPC-BASED DistRibuted CONTROL}

In this section, a PPC algorithm is presented to accurately obtain the global utilization level $K^{U}$ which is further used to update the active power references by (5) and achieve the control objectives.

\section{A. Control Development}

First, rewrite (4) as $K^{U}=\frac{\sum_{i \in \mathcal{V}_{E}} P_{i} / N}{\sum_{i \in \mathcal{V}_{E}} P_{i}^{M} / N}$. Since the numerator and denominator are the average values of the active power outputs and capacities, respectively, they can be obtained through the traditional average consensus algorithm [10].

For clarity, define two states, $\bar{P}_{i}$ and $\bar{P}_{i}^{M}$, for DG $i$, $i \in \mathcal{V}_{E}$. Let $\bar{P}_{i}(0)$ and $\bar{P}_{i}^{M}(0)$ be the actual sampled active power output and capacity data, respectively. Further, denote $\bar{P}_{i}(k)$ and $\bar{P}_{i}^{M}(k)$ as the states of DG $i$ at periodic discretetime instants $t=k T_{C}, k=0,1,2, \ldots$, where $T_{C}$ is the communication period for the distributed controllers. Notice that only the data $\bar{P}_{i}(0)$ and $\bar{P}_{i}^{M}(0)$ are sensitive and should be protected since they contain the actual information of DGs.

For brevity, only the acquirement of the numerator $\sum_{i \in \mathcal{V}_{E}} P_{i} / N$ is presented in detail, and that of the denominator is similar and therefore omitted here. According to [10], the traditional consensus algorithm can be expressed as $\bar{P}_{i}(k+1)=w_{i i} \bar{P}_{i}(k)+\sum_{j \in \mathcal{N}_{i}} w_{i j} \bar{P}_{j}(k)$, where $w_{i j}$ being the Metropolis weights [7], and $\mathcal{N}_{i}$ denotes the set of the neighbors of DG $i$. Notice that at $k=0$, the actual active power output data $P_{j}=\bar{P}_{j}(0)$ of DG $j, j \in \mathcal{N}_{i}$ is directly transmitted to its neighbor DG $i$. To protect the sensitive data 


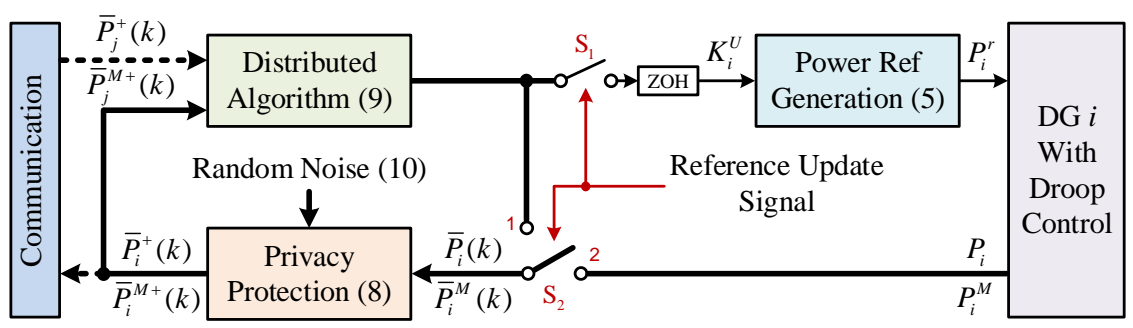

Fig. 2. Diagram of the proposed PPC-based distributed controller.

$\bar{P}_{i}(0)$, a random noise $d_{i}(k)$ is added to the sensitive data before it is sent to its neighbor DGs as follows

$$
\bar{P}_{i}^{+}(k)=\bar{P}_{i}(k)+d_{i}(k) .
$$

The PPC algorithm is then given as

$$
\bar{P}_{i}(k+1)=w_{i i} \bar{P}_{i}^{+}(k)+\sum_{j \in \mathcal{N}_{i}} w_{i j} \bar{P}_{j}^{+}(k) .
$$

As analyzed in Section III, to achieve the control objectives, the accurate value of the utilization level $K^{U}$ is required, i.e., the accurate average values of the active power outputs and capacities are required. For this purpose, the random noise for DG $i, i \in \mathcal{V}_{E}$ can be generated locally by

$$
d_{i}(k)= \begin{cases}r_{i}(k), & k=0 \\ r_{i}(k)-r_{i}(k-1), & \text { others }\end{cases}
$$

where $r_{i}(k)$ is a random number uniformly distributed in the closed set $\left[-\alpha \rho^{k+1}, \alpha \rho^{k+1}\right]$ at each iteration $k$ with $\alpha>0$ and $0<\rho<1$. According to [7, Corollary 3.2], all the states $\bar{P}_{i}$ can converge to the average of their initial values accurately, i.e., $\bar{P}_{i}(\infty)=\sum_{i \in \mathcal{V}_{E}} \bar{P}_{i}(0) / N, i \in \mathcal{V}_{E}$.

Notice that $\bar{P}_{i}(0)$ is equal to the actual active power output $P_{i}$. Hence, $\bar{P}_{i}(\infty)=\sum_{i \in \mathcal{V}_{E}} P_{i} / N$. The average value of the DGs' active power outputs is obtained in a distributed manner with preserved privacy.

Similarly, with the PPC algorithm, the average value of the DGs' active power capacities can be attained, i.e., $\bar{P}_{i}^{M}(\infty)=$ $\sum_{i \in \mathcal{V}_{E}} P_{i}^{M} / N$. Then the local utilization level can be calculated as

$$
K_{i}^{U}=\frac{\bar{P}_{i}(\infty)}{\bar{P}_{i}^{M}(\infty)}=\frac{\sum_{i \in \mathcal{V}_{E}} P_{i} / N}{\sum_{i \in \mathcal{V}_{E}} P_{i}^{M} / N}=K^{U} .
$$

Thus, the global utilization level $K^{U}$ can be acquired accurately through (9) with preserved privacy.

\section{B. Privacy-Preserving Performance Analysis}

In this study, the privacy-preserving performance is defined in the sense of $(\epsilon, \sigma)$-data-privacy, which is expressed as

$$
\operatorname{Pr}\left\{\left|\hat{P}_{i}-P_{i}\right| \leq \epsilon\right\} \leq \sigma
$$

where $\hat{P}_{i}$ is the estimate of $P_{i}, i \in \mathcal{V}_{E}$. The $(\epsilon, \sigma)$-data-privacy indicates that for a given estimation accuracy $\epsilon$, the maximum probability that the difference between the original data and the estimated one is no larger than $\epsilon$ is $\sigma$.

Based on [7, Theorem 3.9] and the condition that $d_{i}(0)=$ $r_{i}(0)$ is uniformly distributed in the closed set $[-\alpha \rho, \alpha \rho]$, the proposed algorithm can achieve the $(\epsilon, \sigma)$-data-privacy with

$$
\sigma=\frac{\epsilon}{\alpha \rho} \text {. }
$$

Hence, combining (12) and (13), one has $\operatorname{Pr}\left\{\left|\hat{P}_{i}-P_{i}\right| \leq\right.$ $\epsilon\} \leq \epsilon /(\alpha \rho)$. Since $\lim _{\epsilon \rightarrow 0} \sigma=0$, the probability of the accurate estimate of $P_{i}$ is 0 . More specifically, the actual information of active power outputs of all DGs can be protected during the discovery process of $K^{U}$. Moreover, with a similar process, the information of actual active power capacities of all DGs can also be protected.

Remark 1: Notice that for a given estimation accuracy $\epsilon$, a larger $\alpha$ can decrease $\sigma$, i.e., the larger the noise, the harder it is for the other DGs to estimate DG $i$ 's $P_{i}$ accurately. However, a larger $\alpha$ will also decrease the convergence speed of the proposed method. In practice, one should properly select $\alpha$ to make a balance between privacy protection and the convergence speed.

\section{Control Implementation}

The control diagram of the proposed PPC-based distributed control design is given in Fig. 2. Firstly, a reference update triggering signal is generated for the microgrid, which is triggered at periodic discrete-time instants $t=0, T_{U}, 2 T_{U}, \ldots$ with $T_{U}$ being the reference updating period. In this study, $T_{U}$ is selected as $T_{U}=k^{\max } T_{C}$ where $k^{\max }$ is the maximum iteration number that can guarantee the convergence of the proposed algorithm.

When the reference update signal is triggered, $S_{1}$ is closed and $S_{2}$ is switched to "2". The outputs of the distributed algorithm, i.e., the average values of the DGs' active power outputs and capacities, are used to calculate the local utilization level $K_{i}^{U}$ through (11). Then the active power references are updated by (5). Besides, the actual active power output $P_{i}$ and capacity $P_{i}^{\max }$ are sampled. To prepare for the next utilization level update, set $k=0, \bar{P}_{i}(0)=P_{i}$, and $\bar{P}_{i}^{M}(0)=P_{i}^{M}$ for the distributed algorithm (9) and the random noise (10).

When the reference update signal is released, $S_{1}$ is open and $S_{2}$ is switched to "1". The local utilization level $K_{i}^{U}$ is maintained unchanged through a zero-order-hold module. The PPC algorithm (9) is implemented to generate a new utilization level $K_{i}^{U}$ for the next updating period. All data being transmitted through the communication network is protected through the privacy protection module with noise added as described in (10).

With the developed power reference generation technique and control structure, according to (5), (6), and (11), $\sum_{i \in \mathcal{V}_{E}} P_{i}=P^{\text {Load }}+P^{\text {Loss }}$ holds every time the active power references are updated. Thus, the supply-demand imbalance that may be caused by using the traditional PPC methods is avoided. 
TABLE I

Microgrid AND CONTROL PARAMETERS

\begin{tabular}{lc|lc}
\hline Quantity & Value & Quantity & Value \\
\hline Nominal frequency $(\mathrm{Hz})$ & 50 & $Z_{i}(\Omega)$ & $2 Z^{b}, 3 Z^{b}, Z^{b}, 4 Z^{b}$ \\
Nominal voltage $(\mathrm{V})$ & 311 & $m_{i}\left(10^{-4}\right)$ & $1.57,2.09,3.14,6.28$ \\
Nominal power $(\mathrm{kW})$ & 10 & $P_{i}^{M}$ (p.u.) & $0.4,0.3,0.2,0.1$ \\
$T_{U}(\mathrm{~ms})$ & 100 & $T_{C}$ (ms) & 4 \\
$w_{12}, w_{23}, w_{34}, w_{41}$ & $1 / 3$ & $\alpha, \rho$ & $5000,0.6$ \\
\hline
\end{tabular}

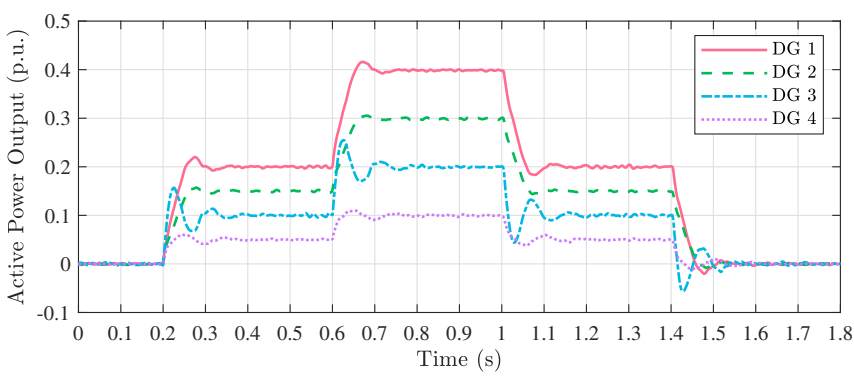

Fig. 3. Active power outputs $P_{i}$ of DGs.

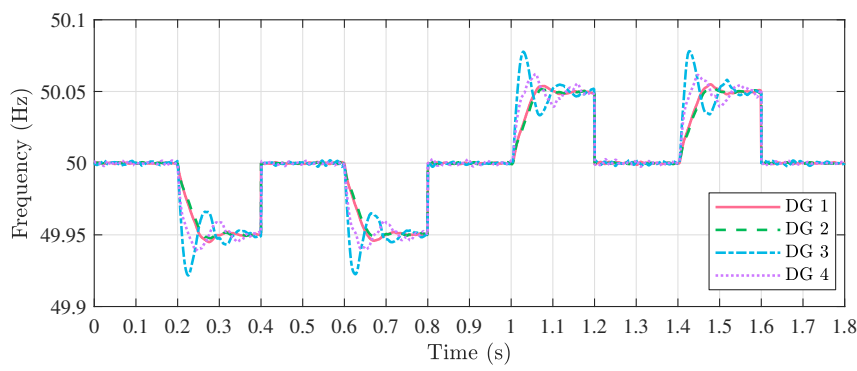

Fig. 4. Frequencies $\omega_{i} / 2 \pi$ of DGs.

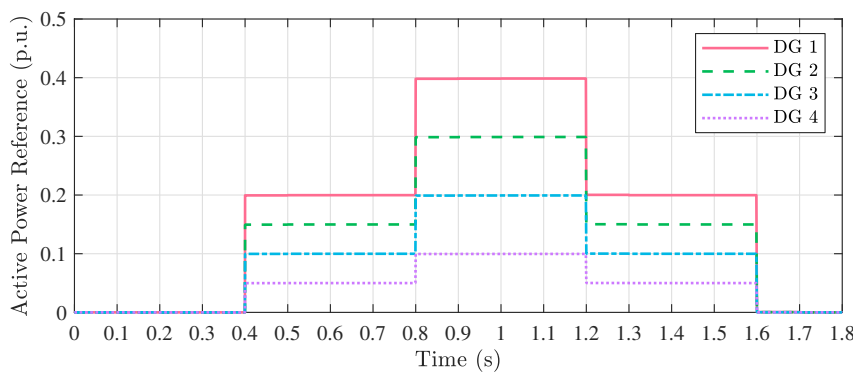

Fig. 5. Active power references $P_{i}^{r}$ of DGs.

\section{VERIFICATION STUDIES}

The performance of the proposed controller is tested with a switch-level microgrid model illustrated in Fig. 1. The system and control parameters are listed in Table I where $Z_{b}=R_{b}+$ $\mathrm{j} \omega L_{b}$ with $R_{b}=0.1 \Omega, L_{b}=1 \mathrm{mH}$. The base power for the calculation of the per unit (p.u.) power value is $10 \mathrm{~kW}$. Two $5 \mathrm{~kW}$ resistive loads are connected to the system at $0.2 \mathrm{~s}$ and $0.6 \mathrm{~s}$, respectively, and disconnected at $1 \mathrm{~s}$ and $1.4 \mathrm{~s}$ afterward.

The corresponding results are shown in Figs. 3-6. From Figs. 4 and 5, one can notice that when the DGs' active power references update at $0.4 \mathrm{~s}, 0.8 \mathrm{~s}, 1.2 \mathrm{~s}$, and $1.6 \mathrm{~s}$, the DGs' frequencies can restore to the nominal value. As shown in Fig. 3, the proportional active power sharing is achieved. Fig. 6 illustrates the comparison results between the sampled power outputs and the power outputs with noise. The transmitted data are totally different from their original ones in both the power outputs and their trends. The privacy of this sensitive

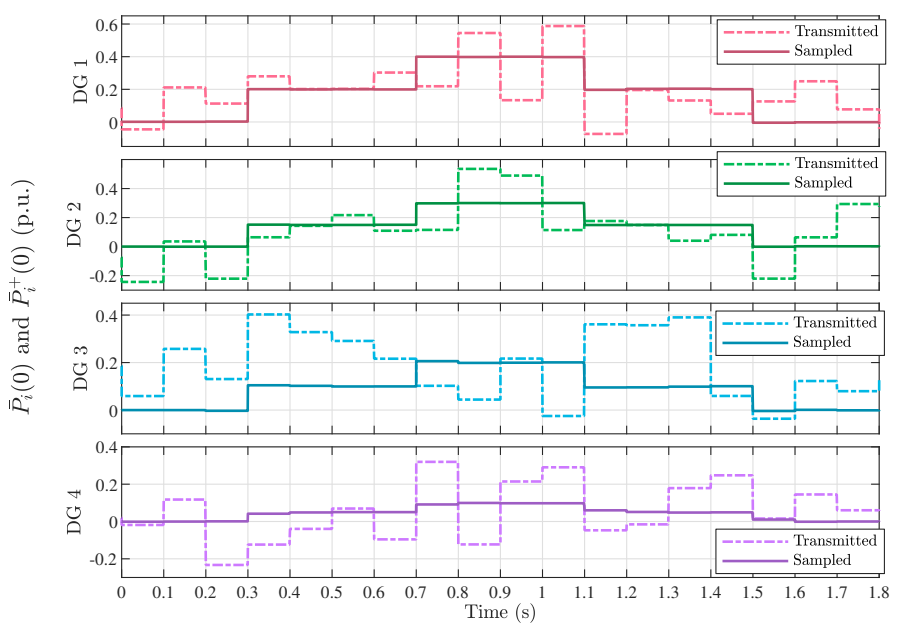

Fig. 6. Comparison between the sampled power outputs $\bar{P}_{i}(0)$ and the transmitted power outputs with noise $\bar{P}_{i}^{+}(0)$.

information is thus protected.

\section{CONCLUSION}

This letter presents a PPC-based algorithm to achieve active power sharing and frequency regulation in microgrids. By virtue of the global utilization level, the original control problem is transformed into an equivalent active power reference generation problem. A PPC-based algorithm is then presented to obtain the global utilization level in a distributed way with preserved privacy. Finally, verification studies are carried out to show the merits of the proposed approach.

\section{REFERENCES}

[1] B. Fan, J. Peng, J. Duan, Q. Yang, and W. Liu, "Distributed control of multiple-bus microgrid with paralleled distributed generators," IEEE/CAA Journal of Automatica Sinica, vol. 6, no. 3, pp. 676-684, 2019.

[2] Y. Khayat, Q. Shafiee, R. Heydari, M. Naderi, T. Dragičević, J. W. Simpson-Porco, F. Dörfler, M. Fathi, F. Blaabjerg, J. M. Guerrero et al., "On the secondary control architectures of AC microgrids: An overview," IEEE Transactions on Power Electronics, vol. 35, no. 6, pp. 6482-6500, 2020.

[3] M. Eskandari, L. Li, M. H. Moradi, P. Siano, and F. Blaabjerg, "Active power sharing and frequency restoration in an autonomous networked microgrid," IEEE Transactions on Power Systems, vol. 34, no. 6, pp. 4706-4717, 2019.

[4] L. Ding, Q.-L. Han, and X.-M. Zhang, "Distributed secondary control for active power sharing and frequency regulation in islanded microgrids using an event-triggered communication mechanism," IEEE Transactions on Industrial Informatics, vol. 15, no. 7, pp. 3910-3922, 2019.

[5] J. Lai, X. Lu, and X. Yu, "Stochastic distributed frequency and load sharing control for microgrids with communication delays," IEEE Systems Journal, vol. 13, no. 4, pp. 4269-4280, 2019.

[6] Q. Zhou, M. Shahidehpour, A. Abdulwhab, and A. M. Abusorrah, "Privacy-preserving distributed control strategy for optimal economic operation in islanded reconfigurable microgrids," IEEE Transactions on Power Systems, vol. 35, no. 5, pp. 3847-3856, 2020.

[7] J. He, L. Cai, P. Cheng, J. Pan, and L. Shi, "Consensus-based dataprivacy preserving data aggregation," IEEE Transactions on Automatic Control, vol. 64, no. 12, pp. 5222-5229, 2019.

[8] T. Yin, Y. Lv, and W. Yu, "Accurate privacy preserving average consensus," IEEE Transactions on Circuits and Systems II: Express Briefs, vol. 67, no. 4, pp. 690-694, 2020.

[9] M. Farrokhabadi, C. A. Cañizares, J. W. Simpson-Porco, E. Nasr, L. Fan, P. A. Mendoza-Araya, R. Tonkoski, U. Tamrakar, N. Hatziargyriou, D. Lagos et al., "Microgrid stability definitions, analysis, and examples," IEEE Transactions on Power Systems, vol. 35, no. 1, pp. 13-29, 2020. 
[10] R. Olfati-Saber, J. A. Fax, and R. M. Murray, "Consensus and cooperation in networked multi-agent systems," Proceedings of the IEEE, vol. 95, no. 1, pp. 215-233, 2007. 